\title{
A study of evacuation efficiency of a hopper-shape exit by using mice under high competition
}

\author{
Lin Peng*, Wang Guoyuan, Wu Fanyu, Gao Dongli \\ Faculty of Geosciences and Environmental Engineering, Southwest Jiaotong University, \\ Chengdu,China \\ Corresponding author. Email: drag76@63.com
}

\begin{abstract}
Exit is the bottleneck of an evacuation from a room and the flow rate through an exit is believed to be depended on its width. A series of experiments were conducted in a bi-dimensional container where mice were driven to pass through two kinds of exit of the identical width, i.e., a conventional exit and a hopper-shape exit. The evacuation efficiency of the two exits was experimentally compared by using mice under competition. The results showed that a hopper-shape exit reduces the escape time by $25 \%$ compared with a conventional exit. Further study was conducted with the presence of a column in front of the two exits. The presence of a column in front of the conventional exit increases the escape time by $22.5 \%$. On the contrary, the placement of column in front of the hopper-shape exit reduces the escape time by $48 \%$. The study showed that the escape efficiency could be greatly improved by appropriately redesigning configuration of exit.
\end{abstract}

Key words: hopper-shape exit, mice evacuation, mean escape time, column in front of an exit

\section{Introduction}

The over-congested crowd, if not managed properly, could lead to disasters[1,2]. For example, 35 people were stampeded to death in the Bund Shanghai on 1st January 2015 and a stampede in Hajj pilgrimage killed around 2177 people on 24th September 2015. Frank and Dorso[3] found the pillarlike obstacle could reduce the evacuation time and the size of the obstacle should be of the order of a pedestrian and the position should be at a distance of 2 or 3 pedestrian sizes. Silo is typical industrial equipment, in which gravity-particles discharge through an outlet. Groups of particles through a narrow exit lead to the spontaneous development of clogs[4]. Full scale experiments $[5,6]$ in silo showed that the presence of an obstacle above the outlet can significantly reduce the clogging probability of granular matter. The physical mechanism behind the clogging reduction is believed to be the obstacles in front of the outlet partially undertake the force of particles, thus the pressure on the outlet decrease significantly. Numerical simulations of gravity-driven granular flow through an hourglass hopper was conducted[7] and it was found that the flow rate across a bottleneck actually increases if an optimized obstacle is placed before it. A number of full-scale experiments [8] by using different inserts in front the outlet were conducted to study their impact on the mass flow and the results suggested that the minimum horizontal distance between the silo hopper and inserts should be twice larger than the silo outlet diameter. Shiwakoti[9-11], Soria[12] and John[13] conducted a series of evacuation experiments using ants as agents. Apart from ants, a group of students were also made as agents in an room evacuation experiment by Garcimartın[14]. Moreover, Garcimartın[15] and Zuriguel[16] presented an experimental study of a flock of sheep passing through a narrow door. Video monitoring of daily routines in a farm was collected by measuring the time lapses between the passages of consecutive sheep, some features of the flow regime can be assessed and the effect of increasing the door size and the performance of an obstacle placed in front of it were evaluated. Lin et al $[17,18]$ and Chen et al[19] conducted a series of experiments by driving the mice to pass through an exit, with or without an obstacle before it. The study shows that the presence of an obstacle in front of an exit can improve or deteriorate the evacuation efficiency depending on the nearby geometry.

In this paper, a series of experiments with mice were conducted at a bi-dimensional space. The objectives of the paper is on one hand, to assess the performance of a hopper-shape exit by comparing with a conventional exit, and to evaluate the impact of column before the two exits on the throughput on the other hand. This paper is structured as follows: the experimental set-up is presented in the subsequent section, and the experimental results will be included in the Section 3. The conclusions will be drawn in Section 4. 


\section{Experimental set-up}

The experiment set-up was introduced by Lin et al [17]. For the completeness, the experimental set-up was only briefly introduced. The experimental setting, as shown in Fig. 1, is composed of two rectangular containers, one is test container for mice evacuation test and the other is rest container to provide food/water/rest for the mice. The two containers are connected by an exit with width of $2 \mathrm{~cm}$. The sizes of the test container are $1.5 \mathrm{~m}$ long, $1.5 \mathrm{~m}$ wide and $2.5 \mathrm{~cm}$ high. The height is only slightly higher than the height of the mice so as to prevent the overlap of mice during evacuation. To increase the competiveness of the exit, the test container is occupied by two blocks with the sizes of $0.6 \mathrm{~m} * 0.3 \mathrm{~m}$, which are inaccessible for the mice. The sizes of the rest container are $0.5 \mathrm{~m}$ long, $1.5 \mathrm{~m}$ wide and $0.3 \mathrm{~m}$ high. The wall and floor of the two containers are made of PVC and the ceiling is made of $6 \mathrm{~mm}$ transparent PMMA for observation during experiment. The column with diameter of D is placed at distances of $\mathrm{L}$ to the exit as shown in Fig.1.

Two kinds of exits, i.e., a conventional exit and a hopper-shape exit, were studied respectively. Conventional exit is the benchmarking study for comparison and hopper-shape exit is the proposed alternative design. For both exits, the exit width was the same at $2 \mathrm{~cm}$. Experimental process was recorded with two digital video cameras. All experiment scenarios are shown in Table 1.

The mice used in the experiment are described as follows: 90 female mice, in the ages of $3 \sim 6$ weeks, their body parameters are: width of $2.8 \sim 3.2 \mathrm{~cm}$, height of $2.3 \sim 2.4 \mathrm{~cm}$, length of $9.5 \sim 10 \mathrm{~cm}$, and weight of $25 \sim 35 \mathrm{~g}$. The mice were trained for weeks to familiar with the location of exit and they escaped toward the exit as far as the burning joss sticks were inserted into Zone A. To produce different levels of stimulus, joss sticks as introduced by Lin et al [17] were used to drive the mice to escape. The experiment showed that when the number of joss stick was increased to 96 , the mice desperately competed with each other and the competition is extreme high. We defined they are in a stressed state. Under this very high smoke concentration, the mice neither lost the direction to escape nor lost their ability to escape.

Table 1. Scenarios of experiment

\begin{tabular}{ccc}
\hline Scenario & Exit Type & Obstacle \\
\hline I & Conventional & No \\
II & Hopper-shape & No \\
III & Conventional & Yes \\
IV & Hopper-shape & Yes \\
\hline
\end{tabular}




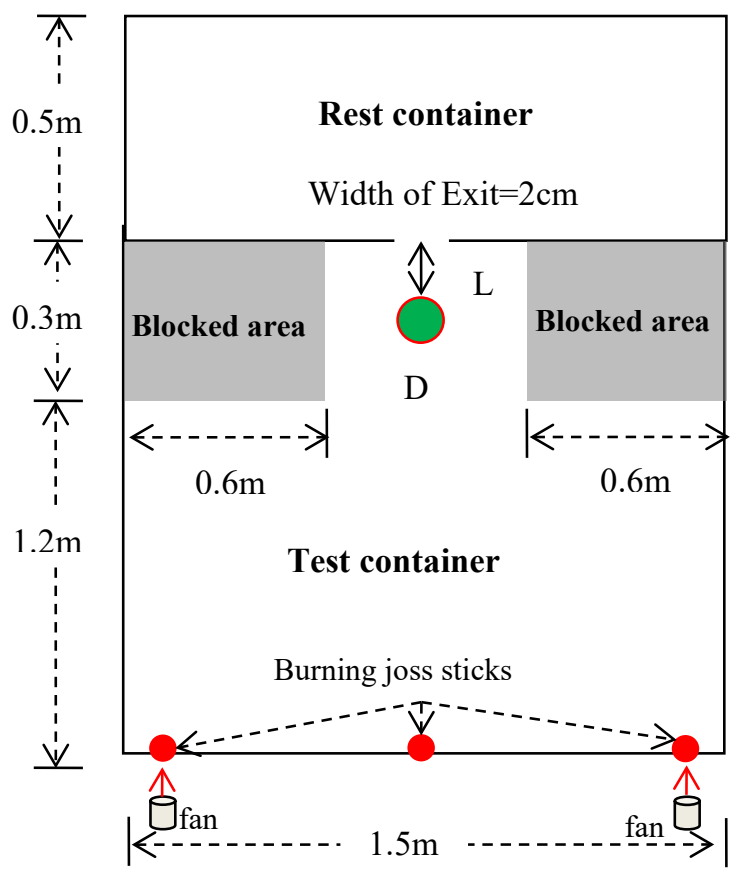

(a) conventional exit

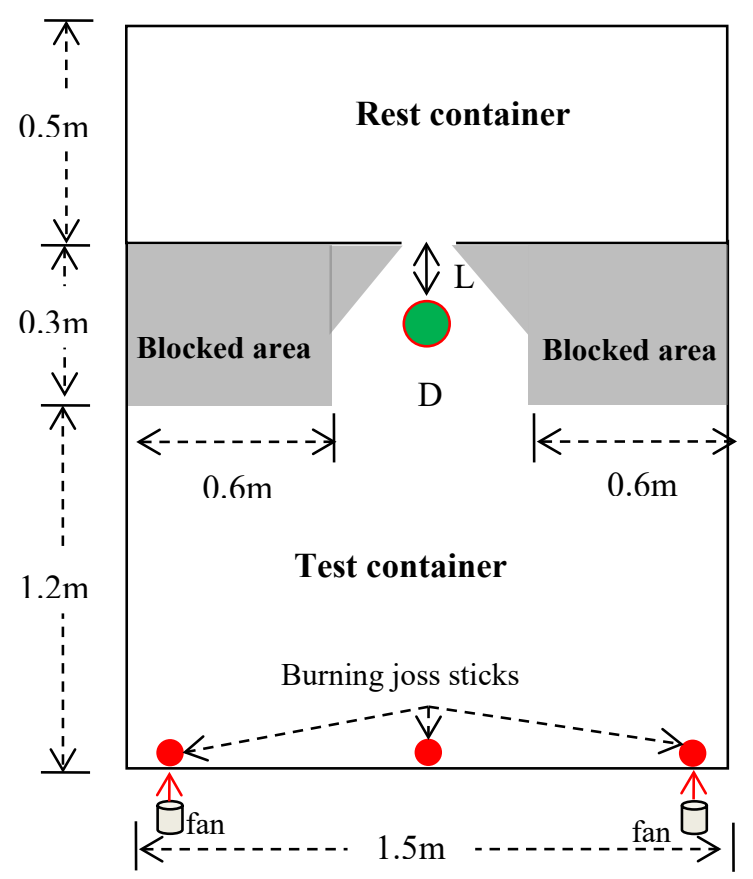

(b) hopper-shape exit

Figure.1 The plan of the experimental set-up (not to scale, the exit width is $0.2 \mathrm{~cm}$ for both exits. The burning joss sticks were placed at three points as indicated. $D$ is the diameter of obstacle, $L$ denotes the distance from the closest boundary of the obstacle to the exit.)

\section{Experimental results}

To minimize the randomness of evacuation process, a number of repeated tests were conducted for each scenario. The number of mice participated in each test varied from 80 95. Each time, a few mice may leave at the test container and only the mice passing through the exit were considered in the estimation of evacuation time. 
In scenario I, the evacuation tests were conducted in the test chamber with conventional exit and the escape times were used as benchmarking for further comparison. The evacuation process is recorded as shown in Fig.2. When the mice were placed into the test container, most of them accumulated at the area close to the exit, others distributed randomly along the boundary of the test container. After the inserting of the burning joss sticks, all mice quickly accumulated at the area close to the exit. 4 repeated tests were carried out and a summary of the results for this situation is included in Table 2 and Fig.3. The time lapses ranged from $1 \mathrm{~s}$ to $20 \mathrm{~s}$ as shown in Fig.3. The mean evacuation time taking for a mouse through the exit was approximately $4.0 \pm 0.4 \mathrm{~s}$. The total evacuation time is not taken as the main measure due to the unstable number of mice passing the exit in different runs of experiment.

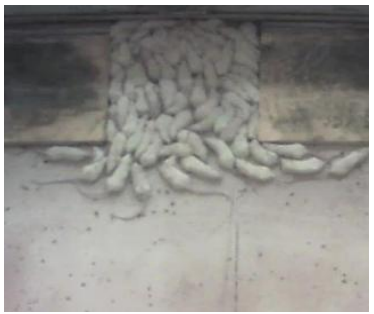

(a) $70 \mathrm{~s}$

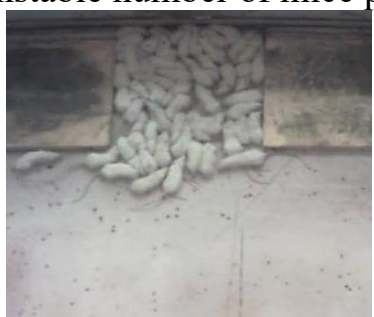

(b) $150 \mathrm{~s}$

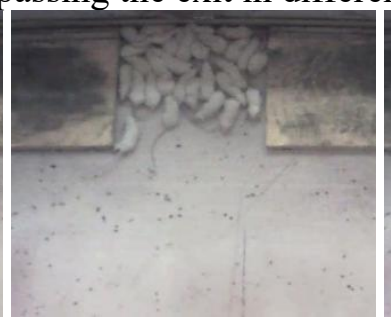

(c) $230 \mathrm{~s}$

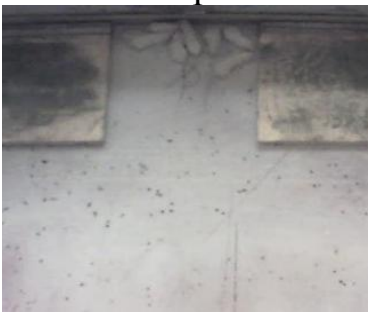

(d)310s

Fig. 2 Recorded escape pattern in scenario I-1

Table 2 Experimental result for scenario I

\begin{tabular}{cccc}
\hline Test No. & $\begin{array}{c}\text { Escape time per mouse } \\
(\mathrm{s})\end{array}$ & $\begin{array}{c}\text { Average evacuation time per mouse } \\
(\mathrm{s})\end{array}$ & $\begin{array}{c}\text { Standard error } \\
(\mathrm{s})\end{array}$ \\
\hline I-1 & 3.9 & & \\
I-2 & 4.0 & 3.7 & 0.3 \\
I-3 & 3.3 & & \\
I-4 & 3.4 & & \\
\hline
\end{tabular}

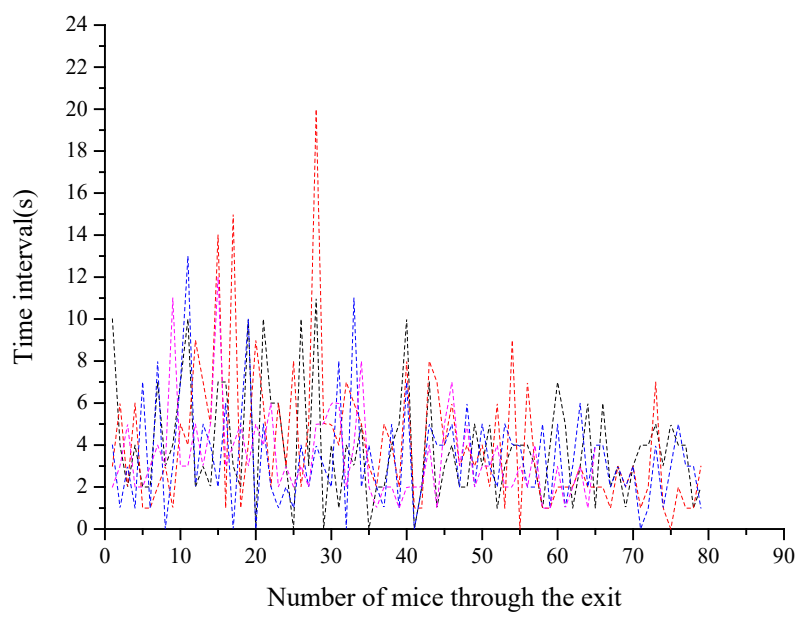

Fig.3. Number of mice through the exit for Scenario I (The dashed lines with different colors as black, red, blue and magenta represents for scenario I-1, I-2, I-3 and I-4 separately.)

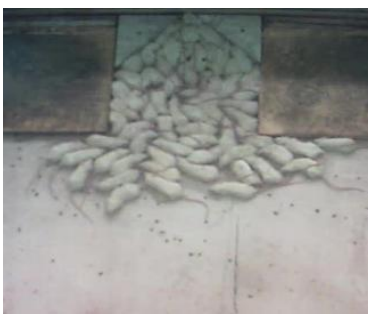

(a) $60 \mathrm{~s}$

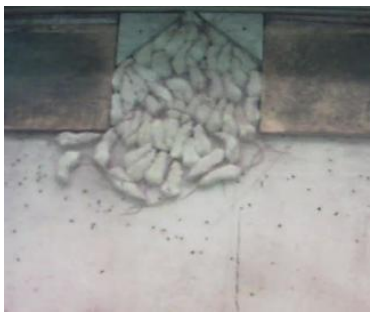

(b) $120 \mathrm{~s}$

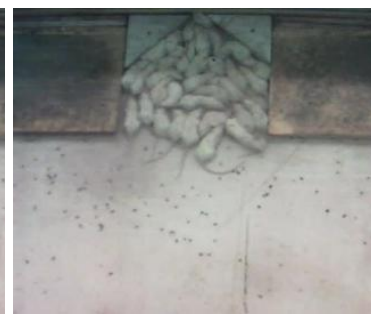

(c) $180 \mathrm{~s}$

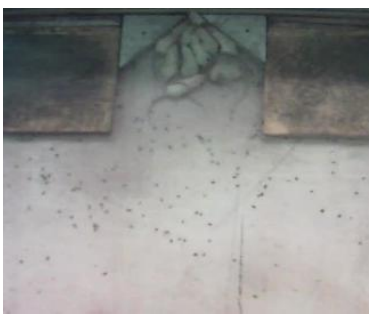

(d) $240 \mathrm{~s}$

Fig. 4. Recorded escape pattern in scenario II-1 
In Scenario II, similar experiments were conducted in the test chamber with the introduction of two triangles as shown in Fig.1(b). The evacuation process is recorded as shown in Fig.4. 4 repeated tests as shown in Table 3. The time lapses ranged from 1s to $18 \mathrm{~s}$ as shown in Fig.5. The mean evacuation time taking for a mouse through the exit was approximately $3.2 \pm 0.5 \mathrm{~s}$. Compared with the results with conventional exit, i.e., Scenario I, the average evacuation time taking for a mouse passing through the exit is reduced by $25 \%$. Clearly, a hopper-shape exit can greatly improve the flow rate.

Table 3. Experimental results for scenario II

\begin{tabular}{cccc}
\hline Test No. & $\begin{array}{c}\text { Escape time per mouse } \\
(\mathrm{s})\end{array}$ & $\begin{array}{c}\text { Average evacuation time per mouse } \\
(\mathrm{s})\end{array}$ & $\begin{array}{c}\text { Standard error } \\
(\mathrm{s})\end{array}$ \\
\hline II-1 & 3.2 & & \\
II-2 & 2.7 & 3.2 & 0.5 \\
II-3 & 3.8 & & \\
II-4 & 3.0 & & \\
\hline
\end{tabular}

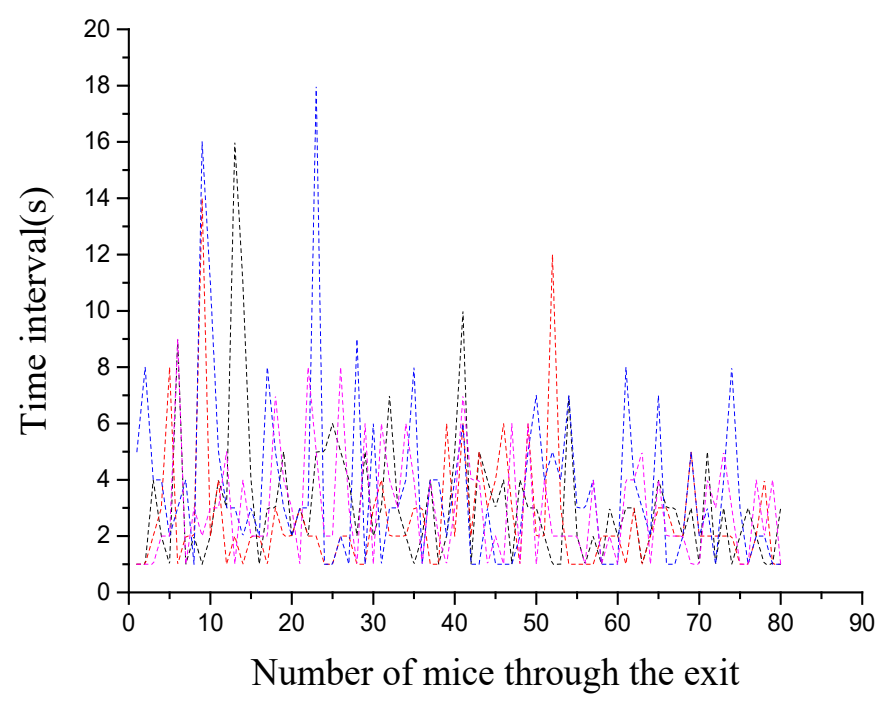

Fig.5. Number of mice through the exit for Scenario II (The dashed lines with different colors as black, red, blue and magenta represents for scenario II-1, II-2, II-3 and II-4 separately.)

Based on previous study in crowds flow, the size of the obstacle is typically of the order of a pedestrian and the position is generally close to the exit (at most at a distance of 2 or 3 pedestrian sizes) and the minimum distance between the silo hopper and column should be twice larger than the silo outlet diameter so as to improve the flow rate. The exit width is $2 \mathrm{~cm}$ and the body width of mouse is around $2.8 \mathrm{~cm} \sim 3.2 \mathrm{~cm}$. Therefore, a column with a diameter of $3.2 \mathrm{~cm}$ were placed at a distance of $2 \sim 4 \mathrm{~cm}$ (around 1 2 times of the exit width) away from the exit. The column was firstly placed in front of the conventional exit at a distance of $2 \mathrm{~cm}$ away from the exit. Experiment was conducted and the results shown that the mice flow was over-crowded due to the presence of the obstacle and the flow was totally clogged. After 10 minutes, less than 20 mice passed through the exit, the experiment was suspended. Two mice close to the exit crushed to death, which lead to the blockage of the exit. A second test was conducted and similar phenomenon was observed and the experiment was suspended after 5 minutes to avoid casualties. Based on initial tests, the presence of column at a distance of $2 \mathrm{~cm}$ (around 1 time of the exit width) cannot improve the flow rate. On the contrary, it significantly reduces the flow rate.

In Scenario III, a column with a diameter of $3.2 \mathrm{~cm}$ was relocated at a distance of $4 \mathrm{~cm}$ away from the conventional exit. The evacuation process is recorded as shown in Fig.6. 4 repeated tests as shown in 
Table 4. The time lapses ranged from $1 \mathrm{~s}$ to $42 \mathrm{~s}$ as shown in Fig.7, which is significantly increased compared with Scenario I. The mean evacuation time taking for a mouse through the exit was approximately

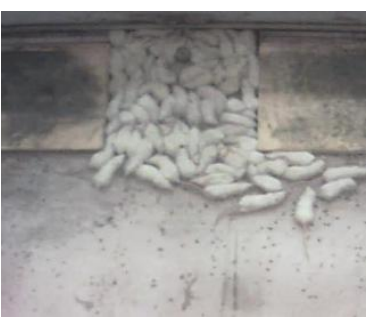

(a) $100 \mathrm{~s}$

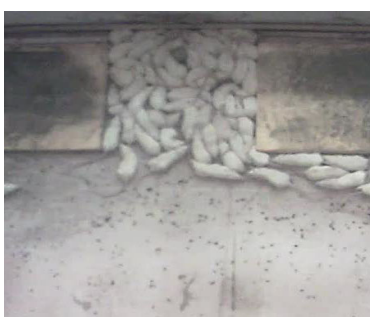

(b) $200 \mathrm{~s}$

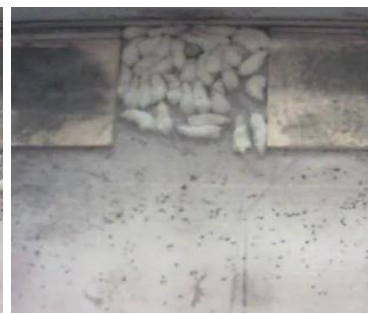

(c) $300 \mathrm{~s}$

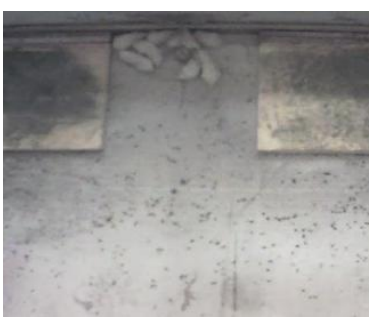

(d) $400 \mathrm{~s}$

Fig. 6. Recorded escape pattern in scenario III-1

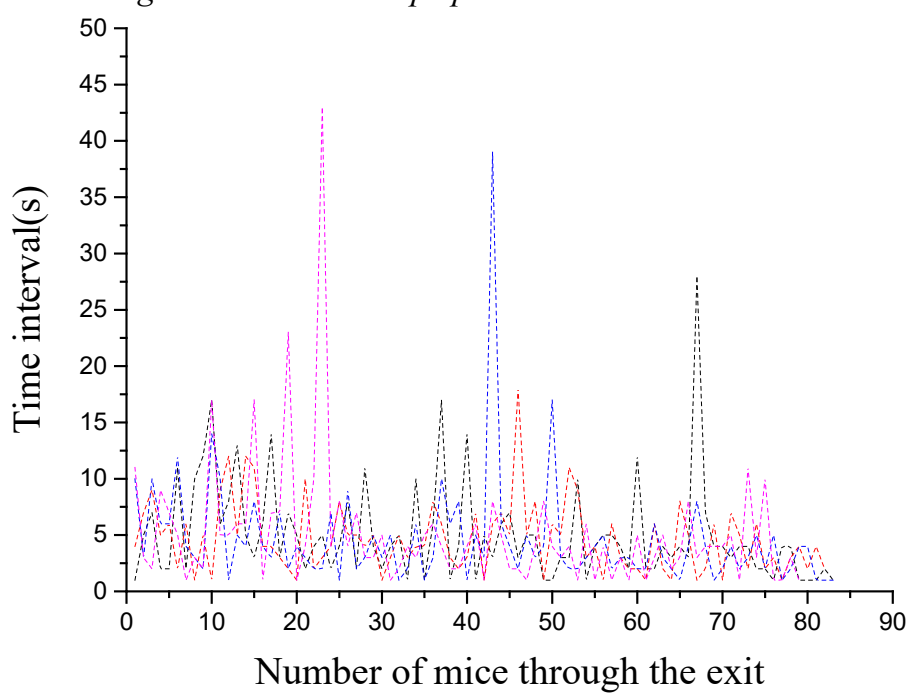

Fig. 7. Number of mice through the exit for Scenario III (The dashed lines with different colors as black, red, blue and magenta represents for scenario III-1, III-2, III-3 and III-4 separately.)

Table 4. Experimental results for Scenario III

\begin{tabular}{cccc}
\hline Test No. & $\begin{array}{c}\text { Escape time per mouse } \\
(\mathrm{s})\end{array}$ & $\begin{array}{c}\text { Average evacuation time per mouse } \\
(\mathrm{s})\end{array}$ & $\begin{array}{c}\text { Standard error } \\
(\mathrm{s})\end{array}$ \\
\hline III-1 & 5.1 & & 0.3 \\
III-2 & 4.6 & 4.9 & \\
III-3 & 4.7 & & \\
III-4 & 5.2 & & \\
\hline
\end{tabular}

Compared with the results in Scenario I, the introduction of an obstacle makes the average evacuation time taking for a mouse passing through the exit increase by $22.5 \%$. The placement of an obstacle in front of conventional exit cannot improve the flow rate. On the contrary, it reduces the flow rate. The result is beyond our previous understanding as we presumed the presence of obstacle should improve the flow rate and reduce the escape time.

In Scenario VI, the column with a diameter of $3.2 \mathrm{~cm}$ was further placed in front of the hopper-shape exit at a distance of $4 \mathrm{~cm}$ above the exit, similar to Scenario III. 4 repeated tests as shown in Table 5 were conducted. The evacuation process is recorded as shown in Fig.8. The time lapses ranged from $1 \mathrm{~s}$ to $12 \mathrm{~s}$ as shown in Fig.9. The mean evacuation time taking for a mouse through the exit was approximately $2.7 \pm 0.3 \mathrm{~s}$. Compared with the results in Scenario II, the mean evacuation time per 
mouse reduces by $18.5 \%$. That is to say, the presence of an obstacle in front of a hopper-shape exit increases the flow rate and the result is basically consistent with the findings in silo flow.

To exploit the flow characteristics of mice flow through the exit at different scenarios, let $\tau$ be the time lapse between two consecutive mice, which reflects the clogging characteristic of mice passing the exit.

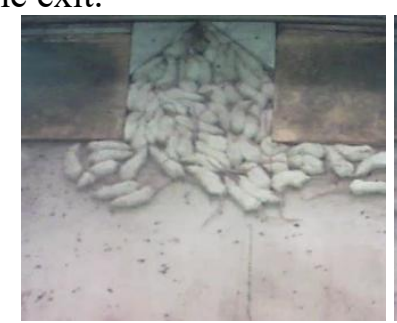

(a) $50 \mathrm{~s}$

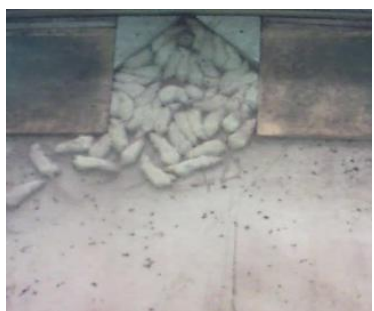

(b) $100 \mathrm{~s}$

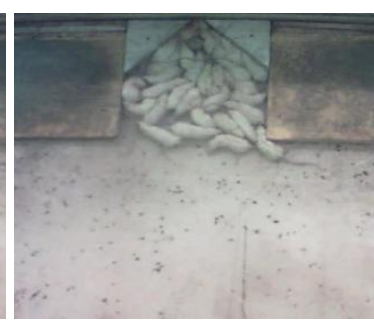

(c) $150 \mathrm{~s}$

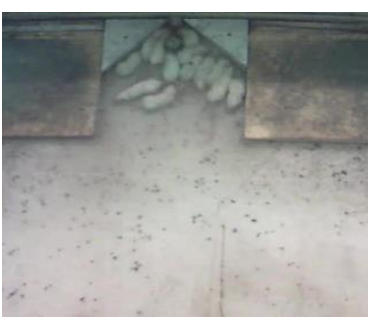

(d) $200 \mathrm{~s}$

Fig. 8 Recorded escape pattern in scenario IV-1

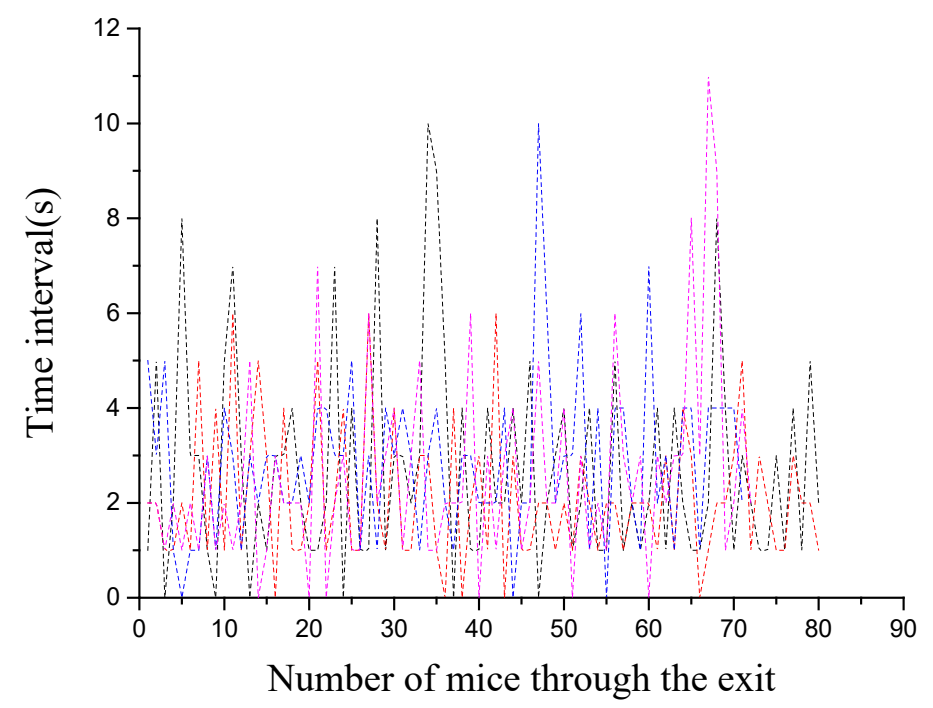

Fig. 9 Number of mice through the exit for Scenario IV (The dashed lines with different colors as black, red, blue and magenta represents for scenario $I V-1, I V-2, I V-3$ and $I V-4$ separately.)

Table 5. Experimental results for scenario IV

\begin{tabular}{cccc}
\hline Test No. & $\begin{array}{c}\text { Escape time per mouse } \\
\text { (s) }\end{array}$ & $\begin{array}{c}\text { Average evacuation time per mouse } \\
(\mathrm{s})\end{array}$ & $\begin{array}{c}\text { Standard error } \\
(\mathrm{s})\end{array}$ \\
\hline IV-1 & 2.8 & & \\
IV-2 & 2.2 & 2.7 & 0.3 \\
IV-3 & 3.0 & & \\
IV-4 & 2.6 & & \\
\hline
\end{tabular}

The time lapses of two consecutive mice for the four scenarios were studied and probability distribution of the time intervals is presented in Fig. 10. The distribution of time lapse follows $P(\Delta t) \sim \tau^{-\alpha}$. Garcimartín[14] suggested that alpha is an indicator of the degree of clogging through the exit and a larger of the Alpha, the smoother of the flow through an outlet. A rigorous mathematical tool as suggested by Clauset et al[20] was used to estimate the alpha for each scenario and the results were presented in Figure 10. 


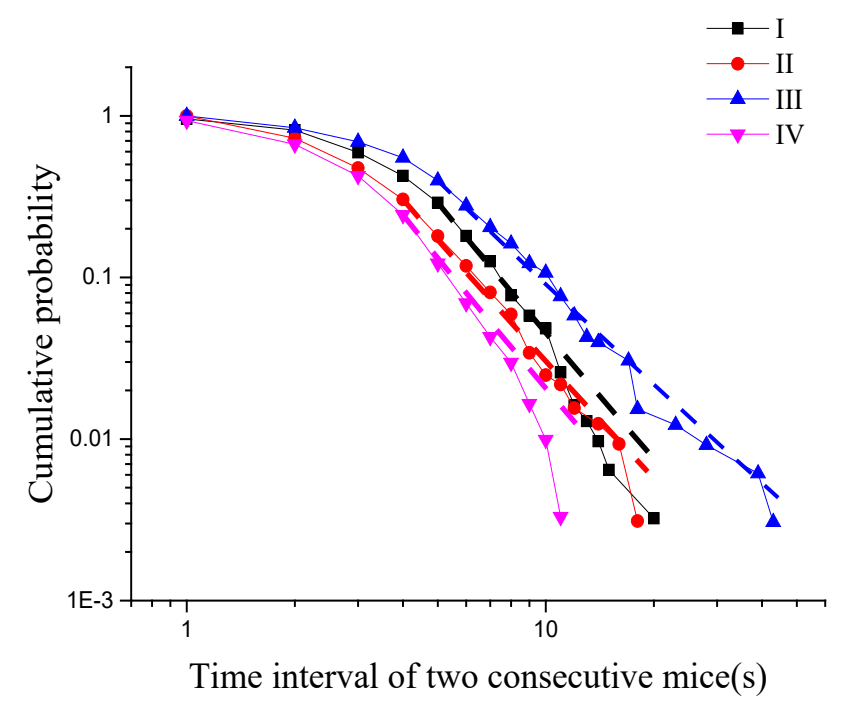

Fig.10 Probability distribution of time intervals (the dashed line is the associated Alpha)

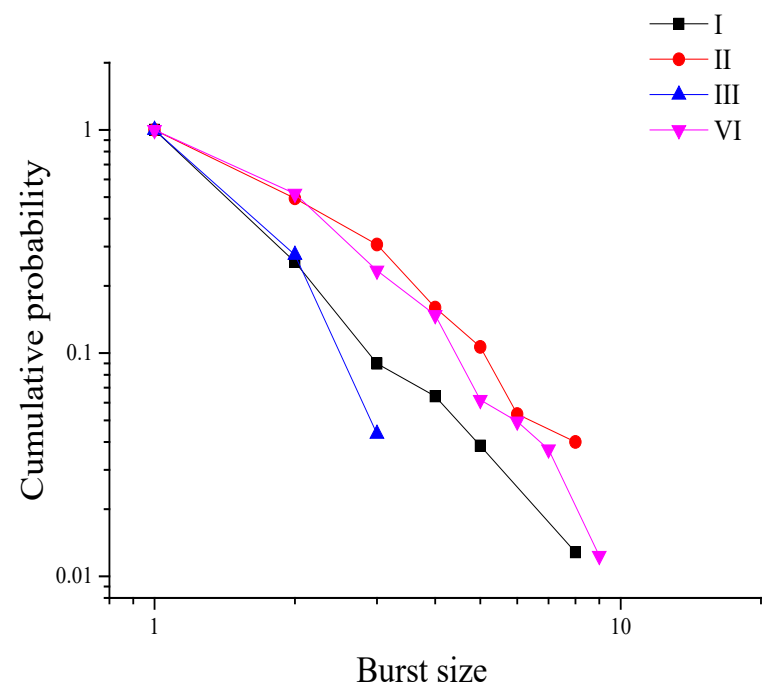

Fig. 11 Probability distribution of burst sizes

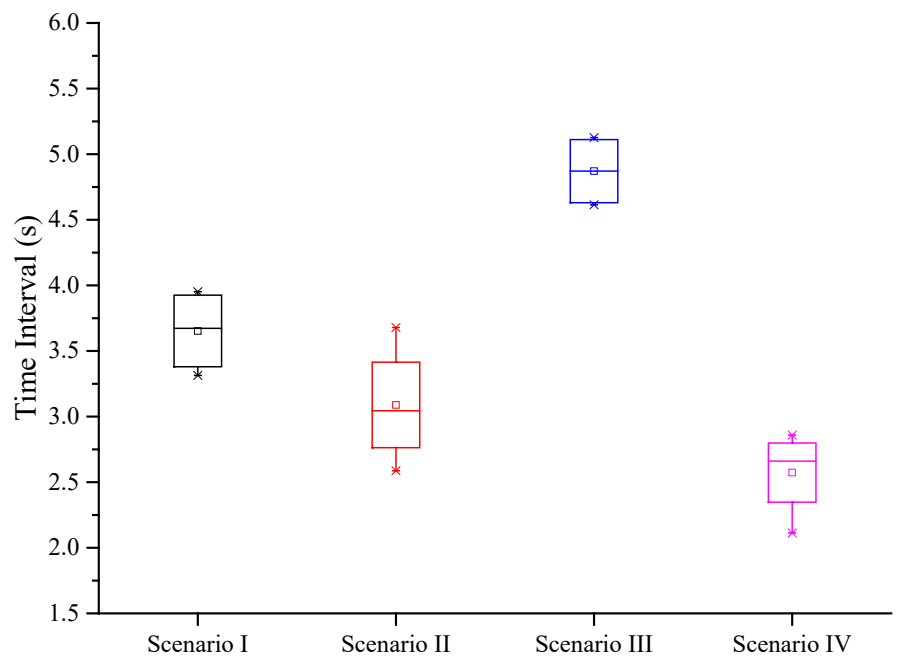

Fig. 12 Mean evacuation time intervals with quartiles of different scenarios 
Alpha $1=3.5$ for scenario I, alpha3=3.5 for Scenario II, alpha2=3.46 for Scenario III, and alpha $4=3.5$ for Scenario IV. No clear relationship between alpha and evacuation efficiency is observed. Our study showed that the alpha is not an indicator of the degree of clogging through the exit. The clogging is defined as no mice passing the exit within 2 seconds. The burst sizes, defined as the continuous number of mice passing through the exit between two clogs, described the properties of intermittent flow through the exit. The burst sizes displayed an exponential behavior as shown in Fig.11.

\section{Discussions and conclusions}

In this paper, a series of experiments with mice under high competition condition were conducted at a bi-dimensional space. The use of mice avoids the ethical and legal issues. To make the test consistency, a group of mice were used in this experiment. The mice were trained a few weeks to learn the skill of evacuation. The evacuation process was video-recorded for further analysis.

Two kind of exits of same width, one is a conventional exit and the other is a hopper-shape exit, were experimentally evaluated by using the mice under strong stimulus. The results are shown in Fig 12, and it is found that the hopper-shape exit reduces the mean escape time by $25 \%$ compared with the conventional exit.

Cohen's d is defined as the difference between 2 means divided by a standard deviation. For scenarios I and II, the value of $d$ is 1.65 , indicating a very large difference between the two scenarios.

The presence of obstacle increase the average evacuation time per mouse by $22.5 \%$ with a conventional exit, whilst the presence of obstacle reduce the average evacuation time per mouse by $18.5 \%$ with a hopper-shape exit. The presence of the obstacle in front of the hopper-shape exit increases the flow rate and this phenomenon agrees with the observation in silo flow. The impact of the obstacle on flow rate through the exit is totally contrary. The value of Cohen's $d$ between scenarios I and III is 4.69, indicating a huge difference between the two scenarios, whilst it is 1.50 between scenarios II and IV, still showing a very large difference.

With a conventional exit, all mice compete to pass through the exit and they are moving in different directions. The strong competition makes the efficiency of exit decreases greatly. With the hoppershape exit, all mice in the space move unidirectionally toward to the exit and the competition at the exit is much attenuated. Therefore, the evacuation time is much reduced. In gravity-driven flow, the driven force is unidirectional. Whilst in mice flow, the driven force is multi-directional. The strong interaction of multi-directional mice flow close to the exit leads to a totally distinctive effect of obstacle on the throughput of exit. In the hopper-shape exit, the mice flow is channeled as unidirectional flow close to the exit, similar to that of silo flow. The interaction of mice is much attenuated as they move in the same direction.

Before the experiment gets started, the mice are trained to get familiar with the position of the exit, so that they would not be wandering in the experiment platform.

In the evacuation experiment conducted by Garcimartín[14], it can be found that the evacuation time interval of competitive evacuees is $0.38 \pm 0.04 \mathrm{~s}$, which is shorter than the mean time interval of mice, and the total evacuation time is nearly $28 \mathrm{~s}$ for 80 evacuees, apparently shorter than that of mice, and the value of the power-law exponent is $a=5.0 \pm 0.1$, which is larger than that of mice. This might due to the limitation of human experiment in which the evacuees are not in an emergency, and that the evacuation could not reflect the phenomenon under dangerous conditions.

Since the hopper-shape exit can improve the evacuation efficiency, it is essential to dig further to find a best angle minimizing the damage caused by emergency, so a series of hopper-shape exit with different angles are going to be tested and analyzed in the future.

\section{Acknowledgement}

The authors greatly acknowledge the valuable comments from anonymous reviewers, the financial support from China Fundamental Research Funds for Central Universities No.2682016cx082 and a number of students who participated the mice experiments. 


\section{References}

[1] D. Helbing, I. Farkas, T. Vicsek, Simulating dynamical features of escape panic, Nature, 66 (2000) 487-490.

[2] A.Johansson, D.Helbing, Analysis of Empirical Trajectory Data of Pedestrians, in: Pedestrian and Evacuation Dynamics 2008, 2010, pp. 203-214.

[3] G.A. Frank, C.O. Dorso, Room evacuation in the presence of an obstacle, Physica A, 390 (2011) 2135-2145.

[4] P. Lin, J. Ma, S.M. Lo, Discrete Element Crowd Model for Pedestrian Evacuation Through an Exit, arXiv:1510.07126v1, (2015).

[5] I. Zuriguel, A.Janda, A.Garcimartı 'n, C.Lozano, R. Are 'valo, D. Maza, Silo Clogging Reduction by the Presence of an Obstacle, Phys. Rev. Lett, 107 ( 2011) 1-5.

[6] L. Celia, J. Alvaro, G. Angle, M. Diego, Z. Iker, Flow and clogging in a silo with an obstacle above the orifice, Phys. Rev. E 86 (2012).

[7] F. Alonso-Marroquin, S.I. Azeezullah, S.A. Galindo-Torres, L.M. Olsen-Kettle., Bottlenecks in granular flow: When does an obstacle increase the flow rate in an hourglass?, Phys. Rev. E, 85, (2012) 020301(R).

[8] J. Tejchman, Confined Granular Flow in Silos Experimental and Numerical Investigations, Springer, 2013.

[9] N. Shiwakoti , M. Sarvi, M. Burd, Using non-human biological entities to understand pedestrian crowd behavior under emergency conditions, Safety Science, 66 (2014) 1-8.

[10] N. Shiwakoti, M. Sarvi, G. Rose, M. Burd, Animal dynamics based approach for modeling pedestrian crowd egress under panic conditions, Social and Behavioral Sciences (2011) 438-461.

[11] N. Shiwakoti, M. Sarvi , G.Rose, M. Burd, Enhancing the safety of pedestrians during emergency egress: can we learn from biological entities?, Transp. Res.Rec, 2137 (2009) 31-37.

[12] S.A. Soria, R. Josens, D.R. Parisi, Experimental evidence of the 'Faster is Slower' effect in the evacuation of ants, Safety Science, 50 (2012) 1584-1588.

[13] A.John, A.Schadschneider, D.Chowdhury, K. Nishinari, Trafficlike collective movement of ants on trails: absence of jammed phase, Physical Review Letters, 102 (2009) 108001.

[14] A. Garcimartín, I. Zuriguel, J.M. Pastor, C. Martín-Gómez, D.R. Parisi, Experimental Evidence of the 'Faster Is Slower' Effect, in: the Conference on Pedestrian and Evacuation Dynamics 2014 (PED 2014), Delft, The Netherlands, 2014, pp. 760-767.

[15] A. Garcimartín, J. M. Pastor, L. M. Ferrer, J. J. Ramos, C. Martín-Gómez, I. Zuriguel, Flow and clogging of a sheep herd passing through a bottleneck, PHYSICAL REVIEW E, 91 (2015) 1-7.

[16] I.Zuriguel, D.R.Parisi, R.C. Hidalgo, C.Lozano, A. Janda, P.A. Gago, Clogging transition of many-particle systems flowing through bottlenecks, scientific reports, 4 (2014) 7324.

[17] P.Lin, J.Ma, T.Y.Liu, T.Ran, Y.L.Si, T.Li, An experimental study of the "faster-is-slower" effect 
using mice under panic, Physica A, 452 (2016) 157-166.

[18] P.Lin, J.Ma, T.Y.Liu, T.Ran, Y.L.Si, F.Y.Wu, G.Y.Wang, An experimental study of the impact of an obstacle on the escape efficiency by using mice under high competition, Physica A, 482 (2017) 228-242.

[19] J. M. Chen, P. Lin, F. Y. Wu, D. L. Gao, G. Y. Wang, Revisit the faster-is-slower effect for an exit at corner, manuscript accepted by

[20] A. Clauset, C.R. Shalizi, M.E.J. Newman, Power-Law Distributions in Empirical Data, SIAM Rev., 51 (2009) 661-703. 